\title{
CORACOID TRANSPLANTATION FOR RECURRING DISLOCATION OF THE SHOULDER
}

\author{
Arthur J. Helfet, Cape Town, South Africa
}

From the Department of Orthopaedic Surgery, Groote Schuur Hospital, and the University of Cape Town

Ten years have passed since the death of W. Rowley Bristow, a leader in orthopaedic surgery and a great clinician. In his memory I present details of an operation which he showed me nineteen years ago and which $I$ have since used exclusively for recurring dislocation of the shoulder.

A happy quality of Rowley Bristow was his ability to reduce fussy surgical problems to simple and logical essentials. This operation typifies this characteristic, for it copes more simply and logically with all the problems of the condition than the fifty-odd operations that have been discarded, and the three or four that are still practised.

Detachment of the glenoid labrum (Bankart 1938) and deformation of the head of the humerus (Hill and Sachs 1940), either singly or together, are the defects that predispose to repeated dislocation. If the glenoid labrum is detached there is nothing to stop the head of the humerus from tipping out of the shallow glenoid fossa into a synovial pouch between the neck of the scapula and the overlying subscapularis muscle. When the posterior surface of the head of the humerus is defective, the normal glenoid labrum is no longer an adequate check and the head luxates into a pouch formed in what Eyre-Brook (1948) called " stretched anterior tissues," and Osmond-Clarke (1948) " a voluminous anterior capsule." There is no mistaking the lax thin-walled anterior capsule. In the first instance, the head takes the gap medial to the glenoid labrum; and in the second, it slips into a pouch lateral to a fixed labrum. The levering force is usually an uncontrolled abduction and lateral rotation movement of the arm. When both defects become established together, the displacements take place more simply and frequently.

Only once has a different mechanism come under my observation. A young soldier reported that for as long as he could remember he had been able actively to displace and reduce the head of his right humerus. And indeed, he could demonstrate the trick with gentle movements of the arm and without lateral rotation. The shoulder had never dislocated involuntarily until he participated in a course of Army physical training. Since then he had lost control and some of the derangements had been painful. Radiographs showed a congenital flattening both of the glenoid fossa and of the medial surface of the head of the humerus. At operation it was realised that he had gradually stretched the anterior structures of the joint and that he was able to glide the flat surfaces on each other in an antero-posterior direction. The glenoid labrum was not detached but was attenuated.

On three occasions cartilaginous loose bodies, about half an inch in length, and presumably fragments of the labrum, were found in the joint. They probably precipitated the particularly painful episodes.

\section{TREATMENT BY OPERATION}

Only surgery offers a remedy for these unstable joints. When the glenoid labrum is detached, whether it is repaired by Bankart's own technique, by "Johannesburg" stapling (du Toit and Roux 1956) or by Eyre-Brook's (1948) method, the results are very good. Grafting a bone block (Palmer and Widén 1948) or a Vitallium prosthesis (Moseley 1953) to the glenoid margin can be most effective in closing the gap. When a humeral head defect and a voluminous anterior capsule are responsible, most successful results are reported from reefing the capsule (Osmond-Clarke 1948) or the subscapularis muscle (Palumbo and Quirin 1950), procedures 
which limit lateral rotation at the shoulder joint, so that the bone defect in the head of the humerus cannot reach the edge of the glenoid. Indeed, when performed by competent surgeons,

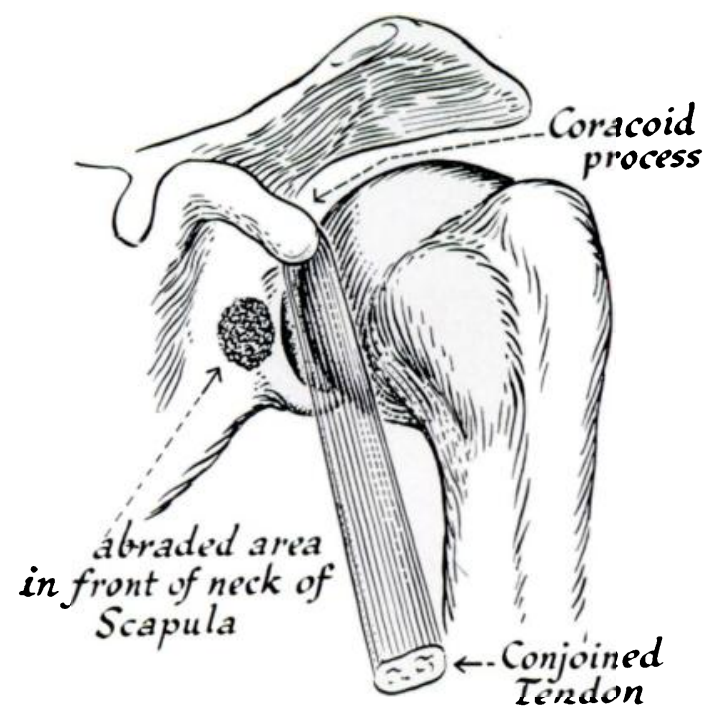

FIG. 1

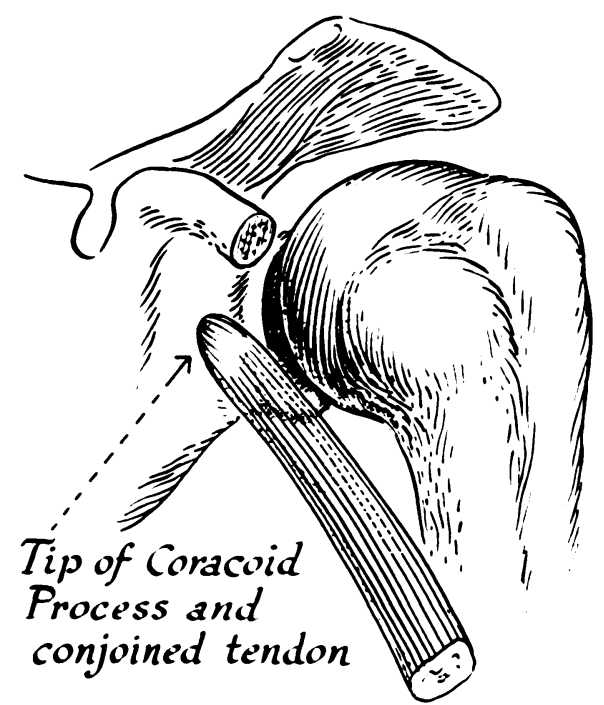

FIG. 2

Diagrams illustrating the principle of the coracoid transplant.

the Putti-Platt and the Bankart operations give results difficult to rival. But they have their technical hazards, for in some hands the joint surfaces are in risk of trauma.

The Bristow operation is logical, is easier to perform and has the added advantage that in the positions of danger - when the arm is abducted and laterally rotated-it becomes more effective.

I present this alternative procedure. therefore, for those surgeons who are not satisfied with their results from other techniques.

The object of the operation is to transplant the terminal half-inch of the coracoid process, which carries the conjoined tendons of the short head of the biceps and the coraco-brachialis, to the neck of the scapula, just medial to the antero-inferior edge of the glenoid rim (Figs. 1 and 2). After six weeks the raw bony surfaces unite either by bone or by firm fibrous tissue. The bone and the tendons form a sound buttress to

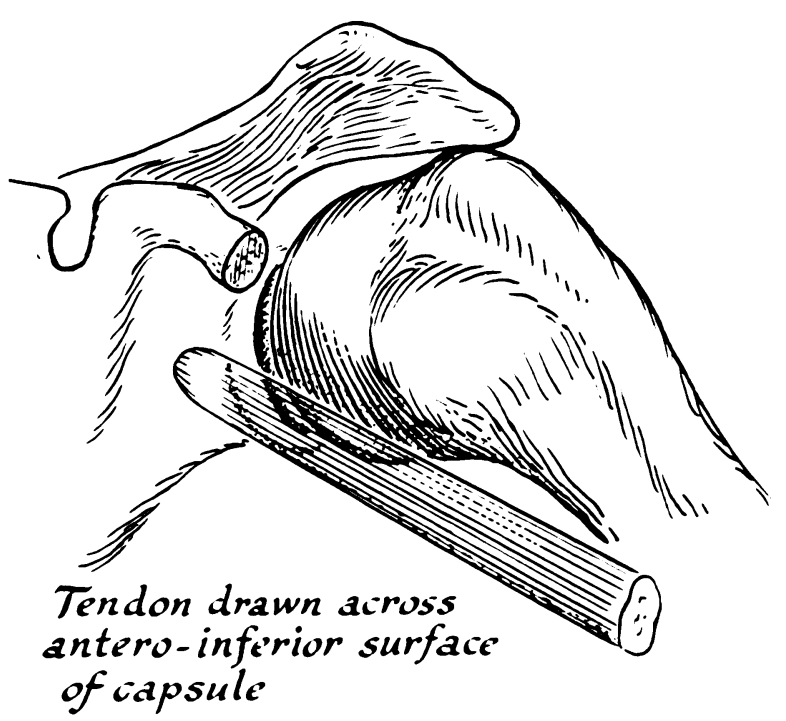

FIG. 3

With the arm abducted and rotated laterally the buttressing action of the transplanted bone and tendons is very effective. the weak part of the capsule. The buttressing effect is increased when the arm is abducted or rotated laterally, because the tendons are drawn more firmly across the antero-inferior part of the capsule (Fig. 3).

\section{TECHNIQUE OF OPERATION}

The shoulder is approached through the usual anterior deltoid incision. The cleft between the pectoralis major and deltoid muscles is widened. Exposure of the coracoid process and of the shoulder joint is facilitated by dividing part of the clavicular origin of the deltoid. 
The coracoid process is divided by bone cutters immediately distal to the insertion of the pectoralis minor. The conjoined tendon is then peeled down gently as far as the entrance of the musculo-cutaneous nerve. There is no need to dissect on the medial side of the conjoined tendon, where the main axillary neurovascular bundle is vulnerable.

The wide insertion of the subscapularis muscle into the lesser tuberosity of the humerus is now displayed (Fig. 4). It is not necessary or advisable to divide the tendon completely.

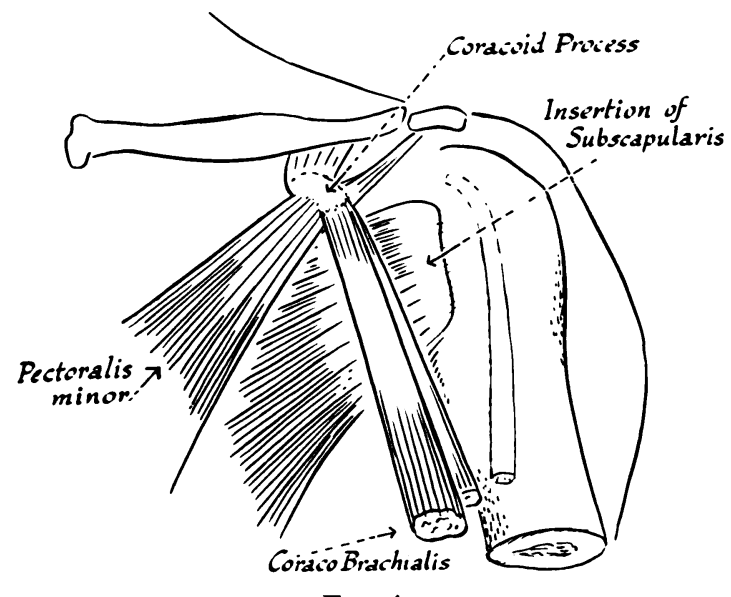

Fig. 4

Technique of operation. Exposure of coracoid process and conjoined tendon.

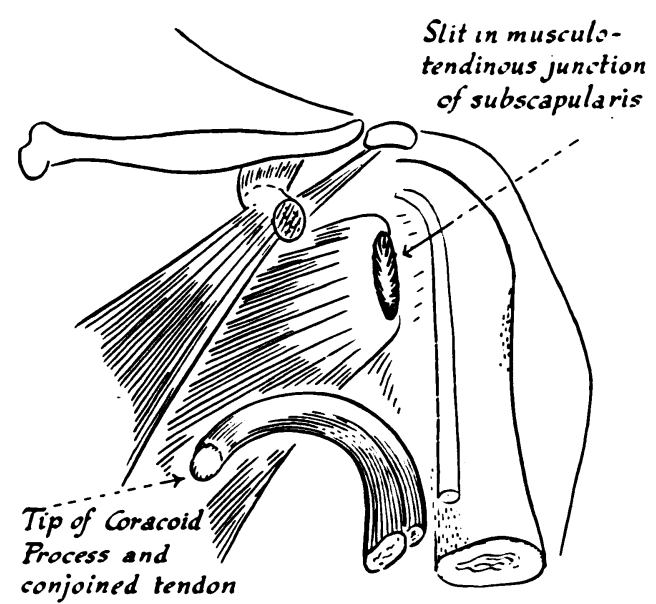

Fig. 5

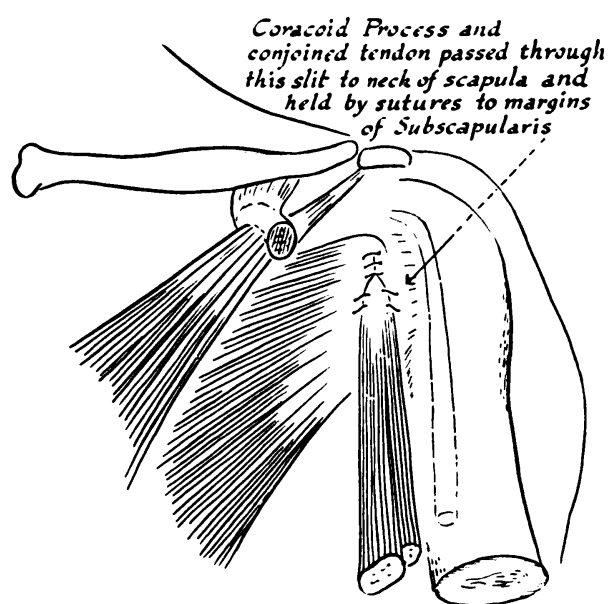

Fig. 6

Technique of operation. Figure 5-The coracoid process has been divided and peeled downwards with the conjoined tendon. A vertical slit has been made through the tendon of subscapularis. The scapular neck is rawed through this slit. Figure 6-The coracoid process has been passed through the slit in the subscapularis, where it lies in contact with the rawed area of the scapula. The subscapularis is closed around the transplanted bone and the attached muscles.

A vertical slit, an inch or an inch and a quarter long, in the middle two-thirds of the musculofibrous junction of the muscle, gives adequate exposure (Fig. 5).

This incision usually opens the joint as well, for the capsule is adherent to the undersurface of the subscapularis. The joint is inspected for loose bodies. The adjacent surfaces of the capsule and subscapularis muscle down to and including the labrum must be dissected apart. The detached labrum and adjacent parts of the capsule are usually found adherent to the subscapularis muscle-an interesting finding that will be commented on later. 
Once the capsule is separated from the subscapularis the bared neck of the scapula adjacent to the naked glenoid rim is exposed. When there is no gleno-labrial detachment the neck of the scapula is clothed with fairly thick periosteal tissue which must be cut and elevated to expose an area of bone about three-quarters of an inch in diameter. The opening in the capsule is sutured, and if the capsule is voluminous it may be overlapped. The surface of the bone on the neck of the scapula is rawed with a fine osteotome or gouge. The coracoid process is passed through the slit in the subscapularis and kept in firm contact with the raw area of bone by incorporating the conjoint tendon in the sutures which approximate the cut edges of the slit in the subscapularis (Fig. 6). The wound is closed.

\section{POST-OPERATIVE CARE}

The arm is supported for six weeks in a collar and cuff sling with a crepe bandage round the arm and trunk to prevent lateral rotation. After a few days, static exercises for the deltoid and upper arm muscles and use of the hand are encouraged. When the limb is released from the bandages, gentle exercises are sufficient to restore movement and power.

\section{COMMENTS}

The effect of the operation is that a block of bone plus the conjoined tendon reinforces the defective part of the joint. It is possible that in some cases the freed glenoid ligament adheres to the rawed bone on the neck of the scapula and so also repairs the Bankart lesion. In abduction and lateral rotation the conjoined tendon is drawn tauter and higher across the antero-inferior surface of the joint, so providing increasing support.

Slight limitation of lateral rotation sometimes results from this operation, but in most cases a full range of movement has been recovered. In view of the increased support provided by the tendons, full movement does not bring any danger of recurrence.

Results-Records of operations in six years of military orthopaedic surgery are no longer available to me, but I recollect only one soldier who could not return to his unit or depot in his original physical category. Reference to this patient has already been made, but whereas before the operation the head of the humerus glided out of the joint, subsequently he felt instability only over that fraction of space while the head moved from the edge of the joint to abut against the bone block. I do not yet know the answer to this particular problem, but the result does demonstrate the "bone block" effect. When there is a glenoid rim and the head must rotate over the edge, the bone block and tendon give full stability.

In Cape Town I have notes of thirty patients. Of these, five boys and one girl, all teenagers, were from a local school for epileptics. One boy redislocated the shoulder in a fit four days after the operation, but further immobilisation brought stability. Another boy, in subsequent fits, developed a posterior dislocation in the same shoulder. Of the other patients only one has suffered a further redislocation. A young athlete who had had a particularly unstable shoulder returned to all his sporting activities after the Bristow operation. One day, a year later, he was angling off the rocks for the big fish that frequent the waters near the Cape of Good Hope. His foot slipped dangerously, and in an endeavour to save himself he dropped his rod and flung up his arm. A searing pain in the shoulder preceded a recurrence of his previous insecurity. Radiographs showed that the coracoid process had been avulsed from a depression in the neck of the scapula by a violent contraction of the conjoined muscles. Operation confirmed this finding and also demonstrated that in this instance the capsule had fused with the bone and insertion of the tendons, thereby repairing the Bankart lesion. When avulsed the bone had dragged the labrum off the bone with it.

Complications-Only one complication has been worthy of note. After release from his bandages, six weeks after operation, one patient found that certain movements produced pain in the distribution of the musculo-cutaneous nerve. Later he developed mild paraesthesia on the outer surface of the upper arm. Exploration of the nerve showed that it entered the 
coraco-brachialis at a rather high level and was vulnerable at the point where the muscle dipped into the slit in the subscapularis. Freeing the nerve at this point relieved his symptoms.

Management of primary dislocations-In view of the fairly general assumption that there is no tendency for the avulsed glenoid labrum to reattach itself to bone, it is interesting that at operation the detached labrum and adjacent capsule are usually found adherent to the subscapularis muscle. Torn ligamentous and capsular tissues tend to repair rapidly and profusely and to form adhesions to all surrounding tissues. In the absence of continuous immobilisation in full medial rotation, and certainly if movement is permitted, the torn edge is nearer the subscapularis and more likely to become attached to it than to the recurrently receding bony surface. Once it is united with the subscapularis there is no hope of normal repair, and moreover lateral rotation of the humerus would increase the gap through which the head luxates. This explanation reinforces Watson-Jones's $(1948,1955)$ adamant advice that acute dislocation should be treated by complete immobilisation in the position of full medial rotation for not less than three weeks.

\section{SUMMARY}

1. Coracoid transplantation for recurring dislocation of the shoulder is described.

2. In my experience of over thirty cases only once has true dislocation recurred after this operation. Recurrence was due to avulsion of the bone block.

3. Failure to repair the original detachment of the glenoid labrum is a frequent cause of recurrence of dislocation of the shoulder. An explanation is offered for this failure; namely that the injured labrum adheres to the deep surface of the subscapularis muscle instead of reattaching itself to bone. This reinforces Watson-Jones's advice that the original dislocation should be treated by complete immobilisation in full medial rotation for three weeks.

The illustrations are by Mr Bruce Franck of Cape Town.

\section{REFERENCES}

Bankart, A. S. B. (1938): The Pathology and Treatment of Recurrent Dislocation of the Shoulder-joint. British Journal of Surgery, 26, 23.

EYRE-BrooK, A. L. (1948): Recurrent Dislocation of the Shoulder. Journal of Bone and Joint Surgery, 30-B, 39. Hill, H. A., and SAChS, M. D. (1940): The Grooved Defect of the Humeral Head. Radiology, 35, 690.

Moseley, H. F. (1953): Shoulder Lesions. Second edition. New York: Paul B. Hoeber, Inc.

OSMOND-Clarke, H. (1948): Habitual Dislocation of the Shoulder. Journal of Bone and Joint Surgery, $30-B, 19$. Palmer, I., and Widén, A. (1948): The Bone Block Method for Recurrent Dislocation of the Shoulder Joint. Journal of Bone and Joint Surgery, 30-B, 53.

Palumbo, L. T., and Quirin, L. D. (1950): Recurrent Dislocation of the Shoulder Repaired by the MagnusonStack Operation. Archives of Surgery, 60, 1,140.

DU ToIr, G. T., and Roux, D. (1956): Recurrent Dislocation of the Shoulder. Journal of Bone and Joint Surgery, 38-A, 1.

Watson-Jones, Sir R. (1948): Note on Recurrent Dislocation of the Shoulder Joint. Journal of Bone and Joint Surgery, 30-B, 49.

WATSON-Jones, Sir R. (1955): Fractures and Joint Injuries. Fourth edition. Vol. 2. Edinburgh and London: E. \& S. Livingstone Ltd. 